\title{
KMT-2018-BLG-0748Lb: sub-Saturn microlensing planet orbiting an ultracool host
}

\author{
Cheongho Han ${ }^{1}$, In-Gu Shin², Youn Kil Jung², Doeon Kim ${ }^{1}$, Jennifer C. Yee ${ }^{3}$, Michael D. Albrow ${ }^{4}$, Sun-Ju Chung ${ }^{2,5}$, \\ Andrew Gould ${ }^{6,7}$, Kyu-Ha Hwang ${ }^{2}$, Chung-Uk Lee ${ }^{2}$, Yoon-Hyun Ryu ${ }^{2}$, Yossi Shvartzvald ${ }^{8}$, Weicheng Zang ${ }^{9}$, \\ Sang-Mok Cha ${ }^{2,10}$, Dong-Jin Kim ${ }^{2}$, Hyoun-Woo Kim ${ }^{2}$, Seung-Lee Kim ${ }^{2,5}$, Dong-Joo Lee ${ }^{2}$, Yongseok Lee ${ }^{2,10}$, \\ Byeong-Gon Park ${ }^{2,5}$, and Richard W. Pogge ${ }^{7}$
}

(The KMTNet Collaboration)

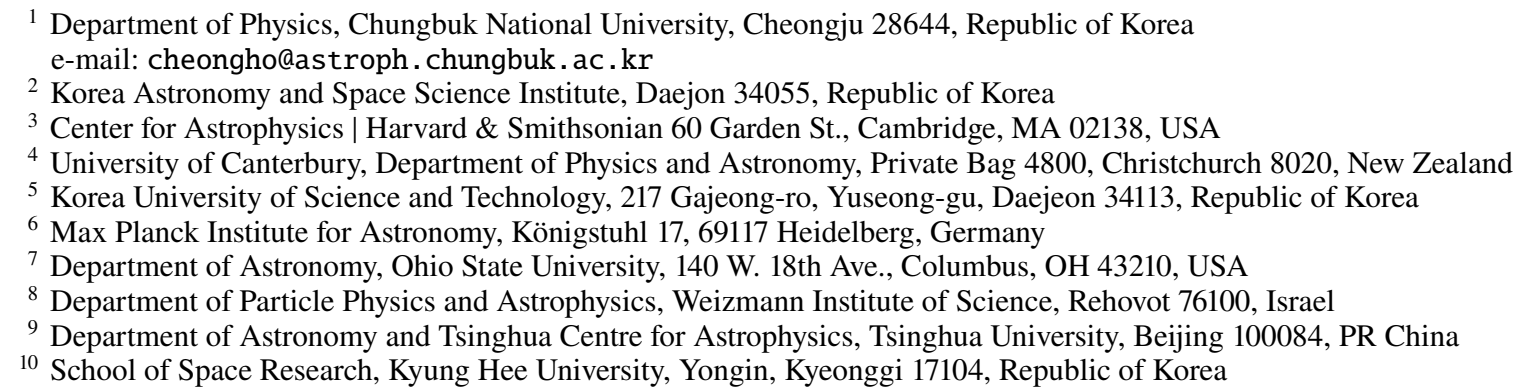

Received 15 April 2020 / Accepted 10 July 2020

\section{ABSTRACT}

Aims. We announce the discovery of a microlensing planetary system, in which a sub-Saturn planet is orbiting an ultracool dwarf host.

Methods. We detected the planetary system by analyzing the short-timescale ( $t_{\mathrm{E}} \sim 4.4$ days) lensing event KMT-2018-BLG-0748. The central part of the light curve exhibits asymmetry due to negative deviations in the rising side and positive deviations in the falling side.

Results. We find that the deviations are explained by a binary-lens model with a mass ratio between the lens components of $q \sim 2 \times 10^{-3}$. The short event timescale, together with the small angular Einstein radius, $\theta_{\mathrm{E}} \sim 0.11$ mas, indicate that the mass of the planet host is very small. The Bayesian analysis conducted under the assumption that the planet frequency is independent of the host mass indicates that the mass of the planet is $M_{\mathrm{p}}=0.18_{-0.10}^{+0.29} M_{\mathrm{J}}$, and the mass of the host, $M_{\mathrm{h}}=0.087_{-0.047}^{+0.138} M_{\odot}$, is near the starbrown dwarf boundary, but the estimated host mass is sensitive to this assumption about the planet hosting probability. High-resolution follow-up observations would lead to revealing the nature of the planet host.

Key words. gravitational lensing: micro - planets and satellites: detection - brown dwarfs

\section{Introduction}

As of the time of writing this article, 107 microlensing planets in 100 planetary systems have been found ${ }^{1}$. Although these microlensing planets comprise a minor fraction of all known planets, microlensing provides an important method to complement other major planet detection methods because of its capability of detecting planets that are difficult to find using other methods. The review paper by Gaudi (2012) describes various advantages of the microlensing method. The microlensing method especially facilitates the discovery of planets orbiting ultracool dwarfs and brown dwarfs (BDs). The most important attribute in order for the microlensing method to have this capability is that planetary microlensing signals rely on the direct gravitational influence of planets and their hosts. As a result, it is not necessary to measure the host flux for the detection of

1 The Extrasolar Planets Encyclopedia (http://exoplanet.eu). a planet, and this enables the method to detect planets that are orbiting very faint hosts and even dark objects.

Detecting planets around ultracool dwarfs is important for various reasons. First, ultracool dwarfs are very common in the Galaxy. According to the present-day mass function (Chabrier 2003), the number density of stars increases down to the lower stellar mass limit of $\sim 0.08 M_{\odot}$. In addition, the extension of the mass function into the BD regime indicates that BD number density is comparable to the stellar number density. Therefore, constructing a planet sample that includes those around ultracool dwarfs is essential to fully census the demographics of planets in the Galaxy. Second, planets around ultracool dwarfs provide a test bed to check the planet formation scenario. Ultracool dwarfs are the lowest mass objects formed through the process of collapsing molecular clouds (Luhman 2012). The low mass of the central object results in the low mass of the accretion disk, and thus the environment of planet formation for ultracool dwarfs would be different from that of regular stars. With a sample of 
planets formed in different environment, it would be possible to probe the planet formation process in limited conditions (Payne \& Lodato 2007).

In this paper, we report a microlensing planetary system, in which a sub-Saturn planet orbits an ultracool dwarf. We present the analysis conducted for the planet discovery according to the following organization. In Sect. 2, we describe the observations of the lensing event from which the planet is detected. In Sect. 3, we describe various models tested for the interpretation of the data. In Sect. 4, we explain the procedure of determining the source type and the angular radius of the Einstein ring. In Sect. 5, we estimate the mass and distance to the lens. A discussion concerning the importance of the discovered planetary system and future follow-up observations to refine the physical lens parameters are given in Sect. 6. A summary of the results and conclusions are presented in Sect. 7.

\section{Data}

The lensing event from which the planetary system is discovered is KMT-2018-BLG-0748. The source (lensed star) of the event lies toward the bulge field at $(\mathrm{RA}, \mathrm{dec})_{\mathrm{J} 2000}=$ $(17: 51: 29.88,-30: 38: 47.00)$, corresponding to $(l, b)=$ $\left(-0^{\circ} .808,-1^{\circ} .974\right)$. The event reached its peak magnification of $A_{\text {peak }} \sim 30$ on $2018-09-10\left(\mathrm{HJD}^{\prime} \equiv \mathrm{HJD}-2450000 \sim 8372\right.$ ). The event duration, defined by the time span of the source brightening beyond the photometric scatter, is about six days.

The event was found by the Korea Microlensing Telescope Network (KMTNet; Kim et al. 2016) survey. The survey commenced its alert-finder system in 2018 (Kim et al. 2018a), but only for a subset of its fields that did not include those containing KMT-2018-BLG-0748. Hence, the event was found from the post-season investigation of the data conducted by Kim et al. (2018b). The event was in the field covered by another lensing survey of the Optical Gravitational Microlensing Experiment (OGLE; Udalski et al. 2015), but no trace of lensing signal was detected because of the source location at the edge of a camera chip.

Although the event lasted a short period of time, the light curve was continuously and densely covered. This coverage was possible for two main reasons. First, the event was observed using multiple telescopes that were globally distributed in three continents. The individual KMTNet telescopes are located at the Siding Springs Observatory (KMTA) in Australia, the Cerro Tololo Inter-American Observatory (KMTC) in South America, and the South African Astronomical Observatory (KMTS) in Africa. The three telescopes are identical with a $1.6 \mathrm{~m}$ aperture. The $9 \mathrm{k} \times 9 \mathrm{k}$ mosaic camera mounted on each telescope yields a very wide field of view of $4 \mathrm{deg}^{2}$, and this enables high-cadence observations of the event. Second, the source is positioned in the overlapping region of the two fields, for which the observational cadence was highest among the total 27 KMTNet fields. The two fields, BLG01 and BLG41, were laid out to overlap each other in most covered area, with a small offset between the fields to cover the gaps among the camera chips. The individual fields were monitored with a 30 min cadence, resulting in a combined cadence of $15 \mathrm{~min}$. Observations were primarily carried out with the $I$ band, and a subset of $V$-band images were acquired to measure the color of the source star.

Photometry of data were conducted via the KMTNet pipeline (Albrow et al. 2009), which utilizes the difference imaging method developed by Tomaney \& Crotts (1996) and Alard \& Lupton (1998). For a subset of the data, we conducted an extra

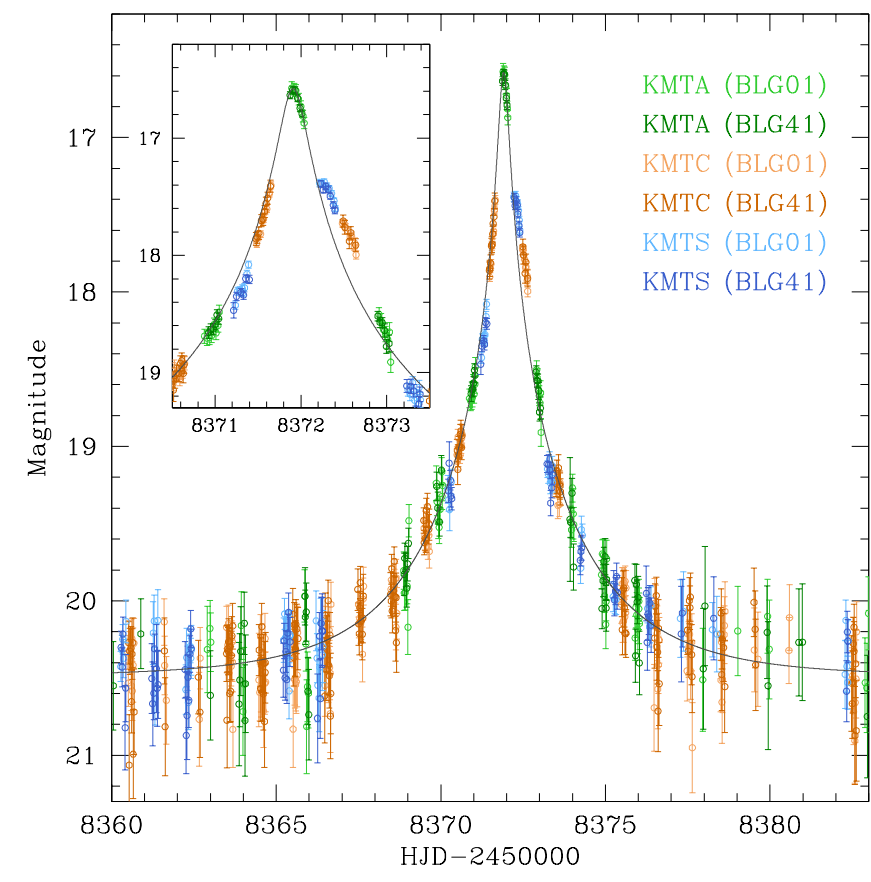

Fig. 1. Light curve of the lensing event KMT-2018-BLG-0748. The zoomed-in view around the peak is shown in the inset. The overplotted curve represents the $1 \mathrm{~L} 1 \mathrm{~S}$ model.

photometry with the pyDIA code (Albrow 2017) to estimate the color of the source star. In Sect. 4, we describe the detailed procedure of estimating the source color. We rescaled the error bars of the data estimated from the photometry pipeline using the method of Yee et al. (2012).

\section{Interpretation of light curve}

\subsection{Single-lens (1L1S) model}

The lensing light curve of KMT-2018-BLG-0748 is shown in Fig. 1. At a casual glance, the light curve seems to be that of a standard event involved with a single lens and a single source ("1L1S"), and thus we first model the light curve under the 1L1S interpretation. Three parameters characterize a 1L1S lensing light curve. These parameters are $t_{0}, u_{0}$, and $t_{\mathrm{E}}$, which represent the time of the minimum lens-source (projected) separation, the separation between the lens and source (scaled to the angular Einstein radius $\theta_{\mathrm{E}}$ ) at $t_{0}$ (impact parameter), and the event timescale, respectively.

The 1L1S model curve is plotted over the data points in Fig. 1. The modeling is conducted so that the peak of the model matches the observed peak at $t_{0} \sim 8371.9$. From the comparison of the observed data with the model, it appears that the observed light curve is approximately described by a 1L1S model with an event timescale of $t_{\mathrm{E}} \sim 4$ days. However, a close inspection reveals that there exist slight deviations from the model, especially in the region around the peak. The inset in Fig. 1 shows the enlarged view of the light curve around the peak.

In order to inspect the detailed structure of the deviations, we display the peak region of the light curve in Fig. 2. We also show the residuals from the $1 \mathrm{~L} 1 \mathrm{~S}$ model in the lower panel. From the inspection of the residuals, it is found that the deviations occur in two major parts. The first part is the region in the rising side of the light curve before the peak during 8371.2 $\lesssim \mathrm{HJD}^{\prime} \lesssim 8371.7$. The data points in this region exhibit negative deviations with 


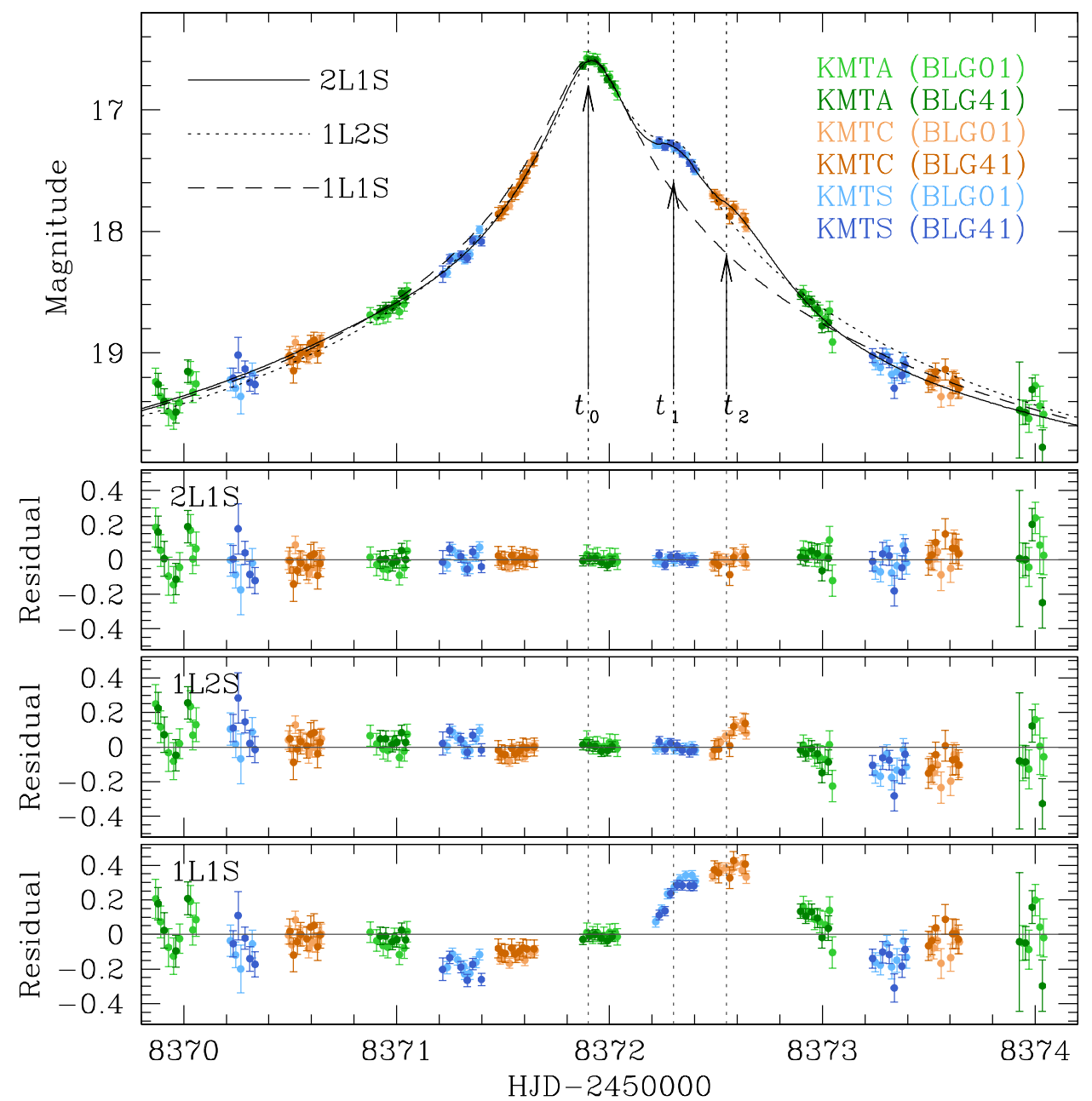

Fig. 2. Various tested models and their residuals. Upper panel: three tested models, 2L1S (solid curve), 1L2S (dotted curve), and 1L1S (dashed curve) models; three lower panels: residuals from the individual models. The three times denoted at $t_{0} \sim 8371.9, t_{1} \sim 8372.3$, and $t_{2} \sim 8372.6$ represent the peak time, and the two bumps, respectively. respect to the $1 \mathrm{~L} 1 \mathrm{~S}$ model. The second part is the region in the falling side after the peak during 8372.2 $\lesssim \mathrm{HJD}^{\prime} \lesssim 8373.0$. In contrast to the first part, the data points in this region exhibit positive deviations. To be noted is that the deviations in this region exhibit two bumps at $t_{1} \sim 8372.3$ and $t_{2} \sim 8372.6$, and the positive deviations are followed by slight negative deviations during $8373.2 \lesssim \mathrm{HJD}^{\prime} \lesssim 8373.5$. The combination of the negative deviations in the rising side and the positive deviations in the falling side makes the light curve appear to be asymmetric.

A lensing light curve may become asymmetric for two major reasons: the binarity of the lens and the binarity of the source. Acceleration of the observer induced by the orbital motion of the Earth can also cause a light curve to appear asymmetric, but for short-timescale events such as KMT-2018-BLG-0748, these microlens-parallax effects (Gould et al. 1994) cannot be the cause of the light-curve asymmetry because the deviation of the source motion from rectilinear during the short duration of the lensing magnification is negligible.

\subsection{Binary-source (1L2S) model}

To investigate the cause of the deviations from the 1L1S model, we first tested a model in which the source is composed of two stars ("1L2S" model). Adding one more source component to the lens modeling required us to include additional parameters together with the $1 \mathrm{~L} 1 \mathrm{~S}$ parameters, that is, $\left(t_{0}, u_{0}, t_{\mathrm{E}}\right)$. Following the parameterization of Hwang et al. (2013), these parameters are $t_{0,2}, u_{0,2}$, and $q_{F}$, representing the peak time and impact
Table 1. Lesing parameters of 1L2S model.

\begin{tabular}{lc}
\hline \hline \multicolumn{1}{c}{ Parameter } & Value \\
\hline$\chi^{2}$ & 1145.8 \\
$t_{0,1}\left(\mathrm{HJD}^{\prime}\right)$ & $8371.918 \pm 0.004$ \\
$t_{0,2}\left(\mathrm{HJD}^{\prime}\right)$ & $8372.336 \pm 0.006$ \\
$u_{0,1}$ & $0.052 \pm 0.002$ \\
$u_{0,2}$ & $0.033 \pm 0.002$ \\
$t_{\mathrm{E}}$ (day) & $3.54 \pm 0.11$ \\
$q_{F}$ & $0.20 \pm 0.02$ \\
\hline
\end{tabular}

Notes. HJD' $=\mathrm{HJD}-2450000$.

parameters of the second source, and the flux ratio of the second source to the first source, respectively. To designate the peak time and impact parameter associated with the primary source, we used the notations $t_{0,1}$ and $u_{0,1}$, respectively. Modeling is done using the initial parameters estimated from the 1L1S modeling and considering the deviation features.

The lensing parameters of the $1 \mathrm{~L} 2 \mathrm{~S}$ model, together with the $\chi^{2}$ value of the fit, are listed in Table 1 . The model curve and residuals from the model are shown in Fig. 2. A comparison of the model fits indicates that the 1L2S model provides a substantially better fit than the 1L1S model by $\Delta \chi^{2}=2739.6$. To inspect how the introduction of a source companion improves the fit, we constructed the cumulative distribution of $\Delta \chi^{2}$ between the 


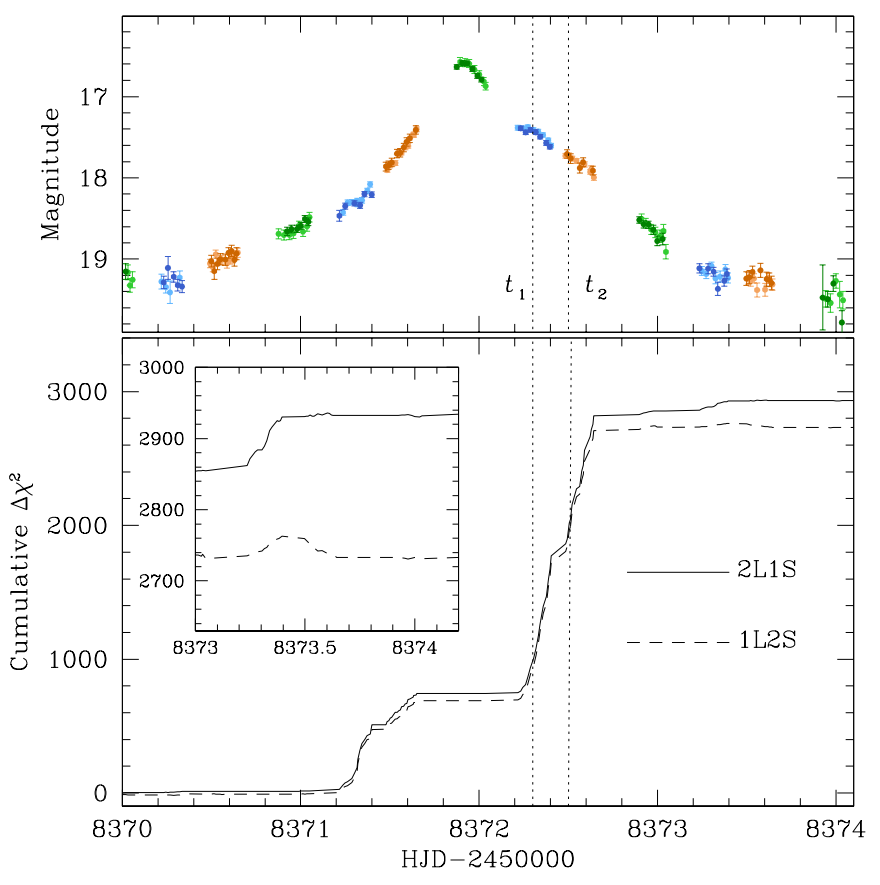

Fig. 3. Cumulative $\Delta \chi^{2}$ distributions of the 2L1S (solid curve) and 1L2S (dashed curve) models with respect to the 1L1S model. The observed light curve is shown in the upper panel to see the locations around which the fit improves. The zoomed-in view of the $2 \mathrm{~L} 1 \mathrm{~S}$ and $1 \mathrm{~L} 2 \mathrm{~S}$ distributions in the region of $8373.0 \lesssim \mathrm{HJD}^{\prime} \lesssim 8374.2$ is shown in the inset of the lower panel. The times denoted by $t_{1}$ and $t_{2}$ corresponds to the times of the two bumps denoted in Fig. 2.

1L2S and 1L1S models and present the distribution in Fig. 3. According to the 1L2S model, the second source, which is fainter than the first source by $\Delta I=-2.5 \log q_{F} \sim 1.75$ magnitude, trails the primary source with a time gap of $\Delta t=t_{0,2}-t_{0,1} \sim 0.42$ days $(\sim 10 \mathrm{~h})$ and approaches the lens with a smaller impact parameter $\left(u_{0,2} \sim 0.033\right)$ than that of the primary source $\left(u_{0,1} \sim 0.052\right)$. We considered finite-source effects to investigate whether the lens passes over the surfaces of the source stars, but we find that the impact parameters of both source trajectories are greater than the source radii, and thus there is no trace of finite-source effects. The introduction of a binary source substantially reduces the negative residuals in the rising side of the light curve. The 1L2S model also reduces the positive residuals in the falling side. However, the model leaves noticeable deviations around the bump at $t_{2}$ and in the negative deviation region during $8373.0 \lesssim$ $\mathrm{HJD}^{\prime} \lesssim 8373.5$.

The validity of the $1 \mathrm{~L} 2 \mathrm{~S}$ model can be additionally checked with the use of the $V$-band data, because the $V$-band data points near the maximum magnification of the second source would manifest a color change (Gaudi 1998; Hwang et al. 2013). Unfortunately, this test cannot be done because the peak region of the second source magnification is sparsely covered by the $V$-band data, and the photometry quality of the few data points is not good enough to securely measure the color change induced by the binary source.

\subsection{Binary-lens (2L1S) model}

We also tested a model in which the lens is a binary (" $2 \mathrm{~L} 1 \mathrm{~S}$ " model). Similar to the 1L2S modeling, considering one more lens component required us to add extra parameters to the lens modeling. These parameters are the $s, q$, and $\alpha$, and they denote
Table 2. Lesing parameters of 2L1S model.

\begin{tabular}{lc}
\hline \hline \multicolumn{1}{c}{ Parameter } & Value \\
\hline$\chi^{2}$ & 937.6 \\
$t_{0}\left(\mathrm{HJD}^{\prime}\right)$ & $8371.901 \pm 0.004$ \\
$u_{0}$ & $0.034 \pm 0.002$ \\
$t_{\mathrm{E}}($ day $)$ & $4.38 \pm 0.15$ \\
$s$ & $0.939 \pm 0.003$ \\
$q\left(10^{-3}\right)$ & $2.03 \pm 0.15$ \\
$\alpha(\mathrm{rad})$ & $-0.023 \pm 0.008$ \\
$\rho\left(10^{-3}\right)$ & $10.89 \pm 0.97$ \\
\hline
\end{tabular}

Notes. HJD' $=$ HJD -2450000 .

the projected separation between the lens components $M_{1}$ and $M_{2}$, the mass ratio (i.e., $q=M_{2} / M_{1}$ ) and the source trajectory angle measured from the $M_{1}-M_{2}$ axis, respectively. A binary lens induces caustics, at which a point-source lensing magnification becomes infinite, and the lensing light curve of a caustic-crossing event deviates from that of a point-source event as a result of the finite-source effect. We considered this finitesource effect in modeling by adding one more parameter of $\rho$, which is defined as $\rho=\theta_{*} / \theta_{\mathrm{E}}$ (normalized source radius). Here $\theta_{*}$ denotes the angular size of the source radius. Finite-source magnifications are computed using the numerical method based on the ray-shooting algorithm (Dong et al. 2006). In this process, we take the surface brightness variation of the source caused by limb darkening into account. We assume that the surface brightness linearly decreases and chose the limb-darkening coefficients considering the spectral type of the source. We discuss the detailed procedure of the source type determination in Sect. 4. With the surface-brightness model $S \propto 1-\Gamma(1-1.5 \cos \psi)$, we adopt $\left(\Gamma_{V}, \Gamma_{I}\right)=(0.62,0.49)$ for the coefficients in $V$ and $I$ bands, respectively. Here $\psi$ represents the angle between the two vectors, which originate at the source center and point toward the observer and toward the source surface.

In the $2 \mathrm{~L} 1 \mathrm{~S}$ modeling, the lensing parameters are categorized into two groups. The parameters $(s, q)$ in the first group are searched for via a grid approach because a lensing magnification can change dramatically with a minor variation of the parameters. For the other parameters, that is, $\left(t_{0}, u_{0}, t_{\mathrm{E}}, \alpha, \rho\right)$, in contrast, the magnification variation is smooth, and thus we look for these parameters using a downhill method based on the Markov chain Monte Carlo (MCMC) algorithm. The grid search for the parameters $(s, q)$ is useful because investigating the $\Delta \chi^{2}$ map on the plane of these parameters enabled us to find possible degenerate solutions, which yield similar models despite the large differences in the lensing parameters. For KMT-2018-BLG-0748, we identified a unique solution without any degeneracy. With the rough position of the local in the $s-q$ plane, we then conducted another modeling to find a refined solution by setting, in this time, all lensing parameter, including $s$ and $q$, as free parameters.

The model curve obtained from the 2L1S modeling and the residuals from the model are shown in Fig. 2. The lensing parameters of the model are presented in Table 2 together with the $\chi^{2}$ value of the fit. To be noted among the parameters is that the estimated value of $q \sim 2 \times 10^{-3}$ is very low, suggesting that the companion to the lens is very likely to be a planet.

We find that the 2L1S model explains the observed light curve well. The relative goodness of the $2 \mathrm{~L} 1 \mathrm{~S}$ model over the other models is shown in the cumulative $\Delta \chi^{2}$ distribution presented in Fig. 3. The $2 \mathrm{~L} 1 \mathrm{~S}$ model improves the fit by 

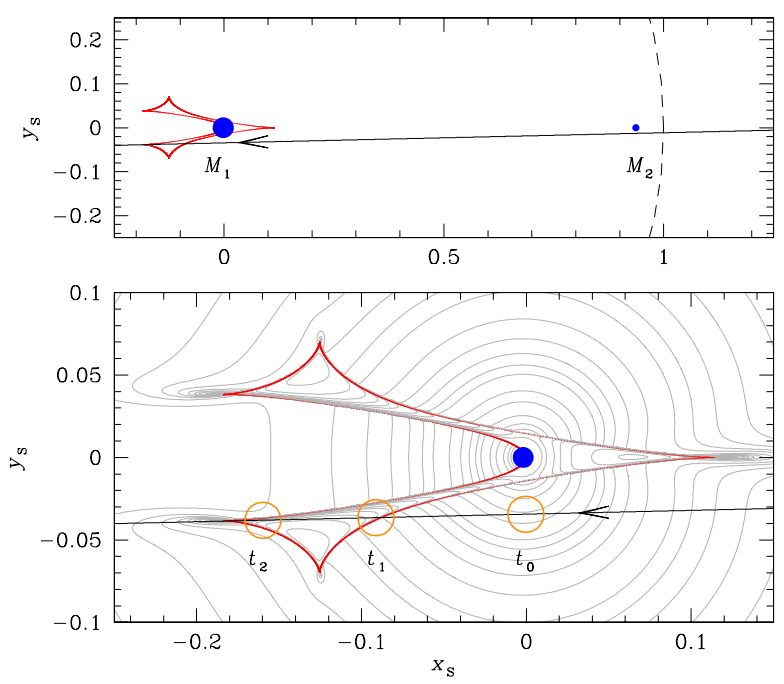

Fig. 4. Configuration of the lens system for the 2L1S solution. Upper panel: zoomed-out region encompassing the binary lens components, the blue dots marked by $M_{1}$ and $M_{2}$; lower panel: zoomed-in view of the central magnification region. The line with an arrow indicates the source trajectory, the cuspy closed curve is the caustic, and the dashed circle in the upper panel represents the Einstein ring. In the lower panel, the three empty circles on the trajectory of the source indicate the source locations corresponding to the times of $t_{0}, t_{1}$, and $t_{2}$ delineated in Fig. 2. The circle size is scaled to $\theta_{\mathrm{E}}$. The gray curves in the lower panel represent equi-magnification contours.

$\Delta \chi^{2}=2947.0$ with respect to the 1L1S model. The model fit is better than that of the 1L2S model by $\Delta \chi^{2}=208.2$, and the residuals from the 1L2S models, that is, the bump at around $t_{2}$ and the negative deviations during $8373.0 \lesssim \mathrm{HJD}^{\prime} \lesssim 8373.5$, vanish. This indicates that the observed deviations are explained by a lens companion rather than a source companion.

The configuration of the lens system according to the $2 \mathrm{~L} 1 \mathrm{~S}$ solution is provided in Fig. 4. In the figure, locations of the binary lens components are indicated by blue dots, the source trajectory is represented by a line with an arrow, and the caustic is shown in red (cuspy closed curve). We note that the separation between $M_{1}$ and $M_{2}, s \sim 0.94$ is similar to $\theta_{\mathrm{E}}$, and this results in a single resonant caustic. The caustic has two prongs extending from the position of the primary lens. The source moves along the path that is nearly parallel to the $M_{1}-M_{2}$ axis, approaching the primary lens, and crossing the lower prong of the caustic two times, that is, caustic entry and exit. The time of the source approach to the primary lens corresponds to the time of the peak magnification, $t_{0}$, and the times of the caustic crossings correspond to the times of the bumps in the light curve at $t_{1}$ and $t_{2}$ indicated by arrows in Fig. 2. A caustic crossing, in general, results in a rapid rising of the lensing magnification, yielding a spike feature in lensing light curves. For KMT-2018BLG-0748, however, finite-source effects are severe, and thus the caustic crossings result in minor bumps instead of spike features. According to the 2L1S model, the slight negative deviations from the 1 L1S model during $8373.0 \lesssim$ HJD' $^{\prime} \lesssim 8373.6$ are explained by the excess magnification extending from the tip of the caustic. We note that these negative deviations and the bump at $t_{2}$ cannot be explained by the $1 \mathrm{~L} 2 \mathrm{~S}$ model.

\section{Angular Einstein radius}

We estimate $\theta_{\mathrm{E}}$ from the measured value of $\rho$ by $\theta_{\mathrm{E}}=\theta_{*} / \rho$. For this, we first estimate $\theta_{*}$ based on the de-reddened color, $(V-I)_{0}$,

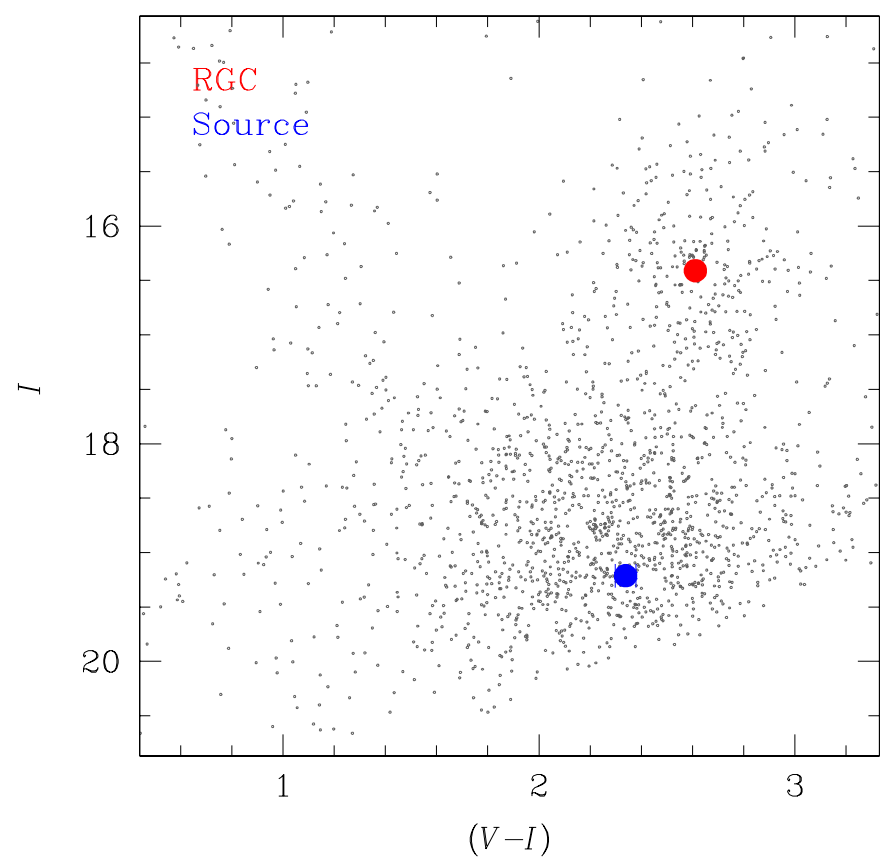

Fig. 5. Color-magnitude diagram of stars located within $2^{\prime} \times 2^{\prime}$ region around the source. The blue and red dots represent the positions of the source and RGC centroid, respectively.

and brightness, $I_{0}$, of the source using the method of Yoo et al. (2004). According to the procedure of this method, we locate the source in the instrumental color-magnitude diagram (CMD), measure the offsets of the source in color, $\Delta(V-I)$, and brightness, $\Delta I$, from the centroid of the red giant clump (RGC), and then estimate $(V-I)_{0}$ and $I_{0}$ using the relation

$(V-I, I)_{0}=(V-I, I)_{\mathrm{RGC}, 0}+\Delta(V-I, I)$.

In this equation, $(V-I, I)_{\mathrm{RGC}, 0}=(1.06,14.47)$ denote the known values of the RGC centroid from Bensby et al. (2013) and Nataf et al. (2013), respectively.

Figure 5 shows the source and RGC centroid in the CMD of stars located in the vicinity of the source. We note that the CMD is produced by conducting photometry on the KMTC data set via the pyDIA software, but the color and brightness are scaled to those of the OGLE-III system (Szymański et al. 2011) to present the calibrated color and brightness. The measured positions of the source and RGC centroid are $(V-I, I)=(2.34 \pm 0.04,19.21 \pm 0.03)$ and $(V-$ $I, I)_{\mathrm{RGC}}=(2.61,16.41)$, respectively. By measuring the offsets of $\Delta(V-I, I)=(-0.27,2.80)$, we estimate that the source has a de-reddened color and a brightness of

$(V-I, I)_{0}=(0.79 \pm 0.04,17.27 \pm 0.03)$.

From the measured color and brightness, it is found that the source is a turn-off star with a spectral type of late G.

The angular radius of the source is deduced from its color and brightness. For this, we first estimate $V-K$ from the measured $V-I$ using the relation between the two colors (Bessell $\&$ Brett 1988), and then estimate $\theta_{*}$ using the relation between $(V-K)$ and $\theta_{*}$ (Kervella et al. 2004). This process yields the source radius of

$\theta_{*}=1.21 \pm 0.10 \mu \mathrm{as}$. 
Table 3. Estimated values of $\theta_{*}, \theta_{\mathrm{E}}$ and $\mu$.

\begin{tabular}{lc}
\hline \hline \multicolumn{1}{c}{ Parameter } & Value \\
\hline$\theta_{*}(\mu \mathrm{as})$ & $1.21 \pm 0.10$ \\
$\theta_{\mathrm{E}}(\mathrm{mas})$ & $0.111 \pm 0.010$ \\
$\mu\left(\mathrm{mas} \mathrm{yr}^{-1}\right)$ & $9.24 \pm 0.80$ \\
\hline
\end{tabular}

With the estimated $\theta_{*}$, the Einstein radius and the relative lenssource proper motion are estimated by

$\theta_{\mathrm{E}}=\frac{\theta_{*}}{\rho}=0.111 \pm 0.013$ mas,

and

$\mu=\frac{\theta_{\mathrm{E}}}{t_{\mathrm{E}}}=9.24 \pm 1.11 \mathrm{mas} \mathrm{yr}^{-1}$,

respectively. The values of $\theta_{*}, \theta_{\mathrm{E}}$, and $\mu$ are summarized in Table 3. Considering that typical lensing events, generated by $\mathrm{M}$ dwarfs located roughly halfway between the observer and source, results in $\theta_{\mathrm{E}} \sim 0.5$ mas, the measured $\theta_{\mathrm{E}}$ value is significantly smaller than those of typical events. Because $\theta_{\mathrm{E}} \propto M^{1 / 2}$, the small value of $\theta_{\mathrm{E}}$ suggests that the lens is a low-mass object.

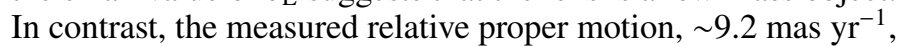
is considerably higher than a typical value of $\sim 5$ mas $\mathrm{yr}^{-1}$.

\section{Physical lens parameters}

The physical lens parameter of the mass, $M$, and distance, $D_{\mathrm{L}}$, are unambiguously determined by measuring the two observables of $\theta_{\mathrm{E}}$ and $\pi_{\mathrm{E}}$, that is,

$M=\frac{\theta_{\mathrm{E}}}{\kappa \pi_{\mathrm{E}}}$

and

$D_{\mathrm{L}}=\frac{\mathrm{au}}{\pi_{\mathrm{E}} \theta_{\mathrm{E}}+\pi_{\mathrm{S}}}$.

In these equations, $\pi_{\mathrm{E}}$ is the microlens parallax, $\kappa=4 G /\left(c^{2} \mathrm{au}\right)$, and $\pi_{\mathrm{S}}$ denotes the parallax to the source (Gould 2000b). Among the two observables needed to determine $M$ and $D_{\mathrm{L}}, \theta_{\mathrm{E}}$ is measured, but $\pi_{\mathrm{E}}$ is not measured in the case of KMT-2018BLG-0748, and this makes it difficult to determine the physical parameters using the relations in Eqs. (6) and (7). We, therefore, estimate $M$ and $D_{\mathrm{L}}$ from a Bayesian analysis using the priors for the Galactic model and lens mass function together with the measured values of $t_{\mathrm{E}}$ and $\theta_{\mathrm{E}}$.

The Bayesian analysis is conducted by generating numerous $\left(4 \times 10^{7}\right)$ artificial lensing events from a Monte Carlo simulation based on the prior models. In the simulation, lenses are physically distributed following a modified Han \& Gould (2003) model and the lens-source transverse speeds of events are assigned based on the modified dynamical distributing model of Han \& Gould (1995). Compared to the original Han \& Gould (2003) model, in which the Galaxy disk is modeled by a simple double-exponential disk, the disk in the modified model has a form of

$\rho_{\text {disk }}=\rho_{\text {disk }, 0}\left[e^{-\left(b^{2}+a^{2} / h_{R+}^{2}\right)^{1 / 2}}-e^{-\left(b^{2}+a^{2} / h_{R-}^{2}\right)^{1 / 2}}\right]$,

by adopting the disk model of Bennett et al. (2014). In this case, $\rho_{\text {disk }, 0}=1.1 M_{\odot} \mathrm{pc}^{-3}$ represents the matter density in the
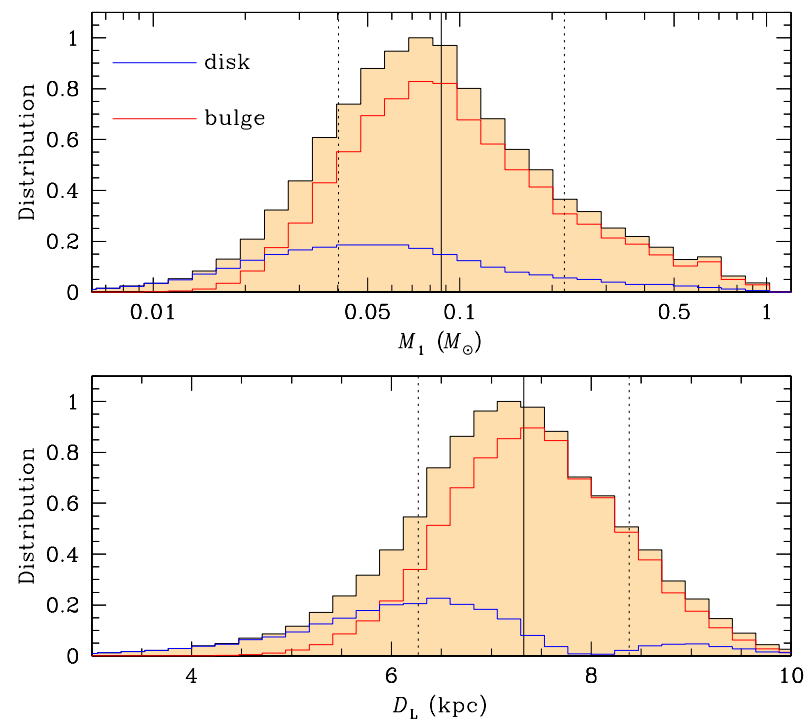

Fig. 6. Bayesian posteriors for the host mass (upper panel) and distance (lower panel) estimated from the Bayesian analysis. In each panel, the blue and red curves indicate the distributions contributed by disk and bulge lenses, respectively, and the black curve indicates that of the two lens populations combined. The solid and dotted vertical lines indicate the median and $1 \sigma$ range, respectively.

solar neighborhood, $a=\left[R^{2}+\left(z / h_{z}\right)^{2}\right]^{1 / 2}, h_{z}=79 \mathrm{pc}, b=0.5$, $\left(h_{R+}, h_{R-}\right)=(2530,1320) \mathrm{pc}$, and $(R, z)$ represent the position in the galactocentric cylindrical coordinates. This modification is done to prevent the increase of the disk density all the way to the Galactic center. The dynamical model of disk objects is modified to account for the change in the matter distribution by changing the velocity dispersions along the $(R, z)$ directions as $\sigma_{R}=$ $\sigma_{R, 0}\left[\Sigma\left(D_{\mathrm{L}}\right) / \Sigma\left(D_{\mathrm{L}}=0\right)\right]^{1 / 2}$ and $\sigma_{z}=\sigma_{z, 0}\left[\Sigma\left(D_{\mathrm{L}}\right) / \Sigma\left(D_{\mathrm{L}}=0\right)\right]^{1 / 2}$, where $\left(\sigma_{R, 0}, \sigma_{z, 0}\right)=(30,20) \mathrm{km} \mathrm{s}^{-1}$ represent the velocity dispersions in the solar neighborhood along the $(R, z)$ directions, respectively. For the bulge velocity dispersions, we used the mean values measured by Gaia. Considering the short timescale and small $\theta_{\mathrm{E}}$ of KMT-2018-BLG-0748, lens masses are assigned using the mass function model of Zhang et al. (2020), which extends down to a substellar mass regime. In the mass function model, we considered remnant lenses, such as white dwarfs, neutron stars, and black holes, by adopting the Gould (2000a) model. We computed the posteriors for $M$ and $D_{\mathrm{L}}$ by obtaining the probability distributions of events with values of $t_{\mathrm{E}}$ and $\theta_{\mathrm{E}}$ located within the uncertainty ranges of the measured $t_{\mathrm{E}}$ and $\theta_{\mathrm{E}}$. We subsequently used the median as a representative value and the 16 and $84 \%$ ranges of the distributions as the uncertainty range of the parameter.

Figure 6 shows the Bayesian posteriors for the primary lens mass $M_{1}$ and $D_{\mathrm{L}}$. In each panel, we present three distributions, in which the blue and red curves are the distributions contributed by disk and bulge lens populations, respectively, and the black curve is the sum of the two lens populations. We find that the disk and bulge contributions are 23 and $77 \%$, respectively. The estimated masses of the primary and companion of the lens are given as

$M_{1}=0.087_{-0.047}^{+0.132} M_{\odot}$,

and

$M_{2}=0.19_{-0.10}^{+0.28} M_{\mathrm{J}}$ 


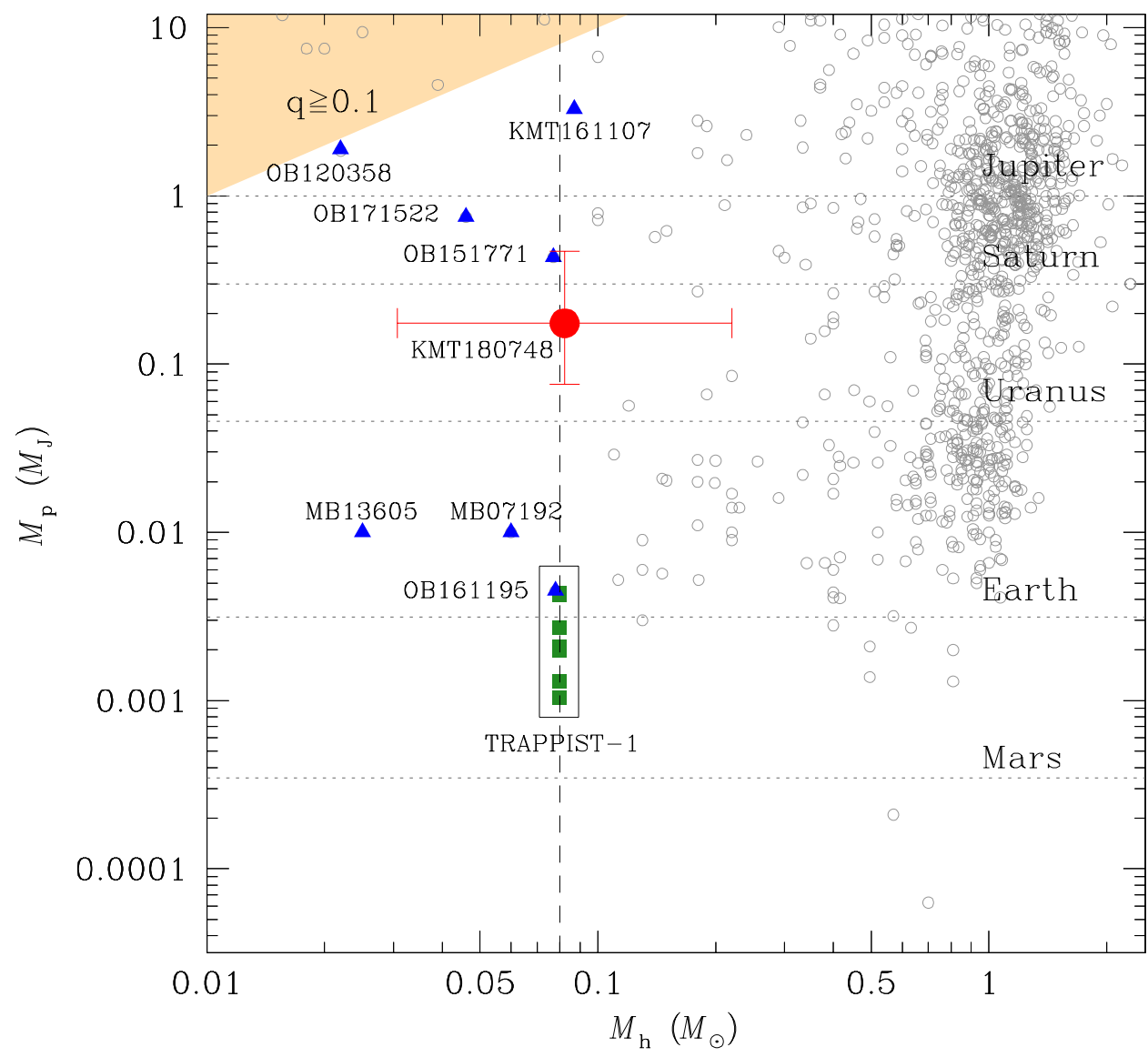

Fig. 7. Distribution of planets in the plane of the host, $M_{\mathrm{h}}$, and planet, $M_{\mathrm{p}}$, masses for planetary systems with known masses. Planetary systems with host masses at around or below the stellar mass limit are denoted by colored points: a red dot for KMT-2018-BLG-0748L, blue triangle dots for the other microlensing planetary systems, and green square dots for the seven planets in TRAPPIST-1 system. The yellow shaded area represents the region with mass ratios $q=M_{\mathrm{p}} / M_{\mathrm{h}} \geq 0.1$. The dashed vertical line represents the star/BD boundary. The dotted horizontal lines represent the masses of the Jupiter, Saturn, Uranus, Earth, and Mars of the solar system from the top to bottom.
Table 4. Physical lens parameters.

\begin{tabular}{lc}
\hline \hline Parameter & Value \\
\hline$M_{1}\left(M_{\odot}\right)$ & $0.087_{-0.047}^{+0.132}$ \\
$M_{2}\left(M_{\mathrm{J}}\right)$ & $0.19_{-0.10}^{+0.28}$ \\
$D_{\mathrm{L}}(\mathrm{kpc})$ & $7.3_{-1.1}^{+1.1}$ \\
$a_{\perp}(\mathrm{au})$ & $0.62_{-0.09}^{+0.09}$ \\
\hline
\end{tabular}

respectively. The planet mass is $\sim 0.63 M_{\mathrm{S}}$ in units of Saturn's mass, and thus the planet is a sub-Saturn planet. We note that the mass of the primary approximately corresponds to the starBD boundary. Considering that the uncertainty of the estimated mass is substantial, the exact nature of the planet host is unclear. The estimated distance to the lens is written as

$D_{\mathrm{L}}=7.3_{-1.1}^{+1.1} \mathrm{kpc}$.

The projected planet-host separation is

$a_{\perp}=s D_{\mathrm{L}} \theta_{\mathrm{E}}=0.62_{-0.09}^{+0.09} \mathrm{au}$.

The planetary separation is much bigger than the snow line of $a_{\mathrm{sl}} \sim 2.7 \mathrm{au}\left(M_{1} / M_{\odot}\right) \sim 0.23 \mathrm{au}$. The estimated physical lens parameters of $M_{1}, M_{2}, D_{\mathrm{L}}$, and $a_{\perp}$ are listed in Table 4.

We note that the estimates of the planet and host masses can vary depending on the assumption about the planet hosting probability. In our analysis, we assume that the planet frequency is independent of the host mass or the planet/host mass ratio. Laughlin et al. (2004) discussed the possibility of the planet frequency dependence on the host mass by pointing out that, within the core accretion paradigm, it would be difficult for giant planets to be formed around low-mass M dwarfs, while such planets would be common around solartype stars. Vandorou et al. (2020) addressed this issue from a microlensing perspective by showing that the host mass of the planet MOA-2013-BLG-220Lb, with a planet/host mass ratio of $q \sim 3 \times 10^{-3}$, determined by analyzing the constraint from high-resolution $\mathrm{AO}$ observations was substantially more massive than the value estimated from the Bayesian analysis conducted under the assumption that stars of all masses were equally likely to host planets of a given mass ratio (Yee et al. 2014). From this result, these authors suggested that planets with planet/host mass ratios $q \sim 2-3 \times 10^{-3}$ might be more likely to be hosted by higher mass hosts. Under this prediction, the mass of KMT-2018BLG-0748L would be more massive than the mass presented in Eq. (9).

\section{Discussion}

The planetary system KMT-2018-BLG-0748L illustrates that microlensing provides an important tool to detect planets orbiting very low-mass stellar and substellar hosts. To demonstrate the importance of microlensing in detecting such planetary systems, in Fig. 7 we present the distribution of planets in the plane of the host, $M_{\mathrm{h}}$, and planet, $M_{\mathrm{p}}$, masses for planetary systems with known masses. There exist nine planetary systems, including KMT-2018-BLG-0748L, with host masses at around or below the star/BD mass limit of $\sim 0.08 M_{\odot}$. These planetary systems include MOA-2007-BLG-192L (Bennett et al. 2008; Gould et al. 2010; Kubas et al. 2012), OGLE2012-BLG-0358L (Han et al. 2013), MOA-2013-BLG-605L 
(Sumi et al. 2016), OGLE-2015-BLG-1771L (Zhang et al. 2020), OGLE-2016-BLG-1195L (Bond et al. 2017; Shvartzvald et al. 2017), KMT-2016-BLG-1107 (Hwang et al. 2019), OGLE-2017BLG-1522L (Jung et al. 2018), and TRAPPIST-1 (Gillon et al. $2016,2017)^{2}$. We indicate these planetary systems with colored points: a red dot for KMT-2018-BLG-0748L, blue triangle dots for the other microlensing planetary systems, and green square dots for the seven planets in TRAPPIST-1 system. Among these systems, eight were detected using the microlensing method except for TRAPPIST-1, which was discovered using the transit method.

We note that the exact nature of the planet host can be identified from future high-resolution follow-up observations. Considering that the mass of the planet host lies at around the star-BD boundary and the uncertainty of the estimated mass from the Bayesian analysis is large, it is not clear whether the host is a low-mass star or a BD. Revealing the nature of the host is possible if the lens is resolved from the source by conducting high-resolution follow-up observations. KMT-2018BLG-0748 is an appropriate target for such observations because the relative proper motion, $\mu \sim 9.2$ mas $\mathrm{yr}^{-1}$, is substantially higher than typical lensing events. Bennett et al. (2007) suggested the possibility of detecting the lens and source from follow-up observations at high resolution using a space-based instrument and this was realized by the detection of the lens star for the planetary microlensing event OGLE-2005-BLG-169 from the Hubble Space Telescope observations conducted when the lens-source separation was $\sim 49$ mas (Bennett et al. 2015). The lens of the same event was also detected from the groundbased AO observations using the Keck telescope conducted by Batista et al. (2015). Observations by the James Webb Space Telescope (JWST) would be able to probe much lower masses than observations by existing $\mathrm{AO}$ systems because the sensitivity of ground-based observations is limited by the bright sky background in the infrared. By implementing a criterion of $\sim 50$ mas separation for the lens and source resolution, the lens of KMT2018-BLG-0748 could be resolved $\sim 5.4 \mathrm{yr}$ after the discovery of the event, that is, in early 2024 .

However, in the case of KMT-2018-BLG-0748, we should adopt a significantly more conservative approach to future AO observations so that a non-detection would be unambiguously interpreted as due to the host being a substellar object. That is, first, we should conservatively estimate the proper motion as $\mu>7$ mas $\mathrm{yr}^{-1}$. And second, we should make allowances for the possibility of a large flux ratio of the lens to source due to the ultracool nature of the first, and so require $\Delta \theta>70$ mas. Thus, to confidently exclude stellar-mass lenses in the event of a non-detection requires waiting $\Delta t=\Delta \theta / \mu>10 \mathrm{yr}$, that is, until 2028.

\section{Conclusions}

The lensing event KMT-2018-BLG-0748 was analyzed and the results from the analysis were presented. For the event, the central part of the light curve appeared to be asymmetric with respect to the $1 \mathrm{~L} 1 \mathrm{~S}$ model due to the negative deviations in the rising side and the positive deviations in the falling side. We tested various models and found that that the deviations were explained by a binary-lens model with a mass ratio between the components of $q \sim 2 \times 10^{-3}$. The small angular Einstein radius,

\footnotetext{
2 We exclude systems with $q \gtrsim 0.1$ because the large mass ratios suggest that they are more likely to form via the mechanism similar to that of binary stars rather than that of planetary systems (Lodato et al. 2005).
}

$\theta_{\mathrm{E}} \sim 0.11$ mas, indicated that the mass of the planet host was very low. From the Bayesian analysis conducted under the assumption of no dependence of the planet frequency on the host mass, it was estimated that the planet had a mass $M_{\mathrm{p}}=0.18_{-0.10}^{+0.29} M_{\mathrm{J}}$ and the mass of the host, $M_{\mathrm{h}}=0.087_{-0.047}^{+0.138} M_{\odot}$, was at around the star/BD boundary, but the host mass would vary depending on the assumption about the planet host probability. Resolving the lens and source would be possible in 2028, provided that the lens is luminous. In that case, the lens mass will be determined from these observations. If the measurement fails to detect the lens, this will imply that the lens is nonluminous and therefore, almost certainly, substellar. In either case, these observations would resolve the nature of the planet host.

Acknowledgements. Work by C.H. was supported by the grants of National Research Foundation of Korea (2017R1A4A1015178 and 2019R1A2C2085965). Work by A.G. was supported by JPL grant 1500811 . This research has made use of the KMTNet system operated by the Korea Astronomy and Space Science Institute (KASI) and the data were obtained at three host sites of CTIO in Chile, SAAO in South Africa, and SSO in Australia.

\section{References}

Alard, C., \& Lupton, R. H. 1998, ApJ, 503, 325

Albrow, M. 2017, MichaelDAlbrow/pyDIA: Initial Release on Github, Version v1. 0.0, Zenodo, http: //dx. doi.org/10.5281/zenodo. 268049 Albrow, M., Horne, K., Bramich, D. M., et al. 2009, MNRAS, 397, 2099 Batista, V., Beaulieu, J.-P., Bennett, D. P., et al. 2015, ApJ, 808, 170 Bennett, D. P., Anderson, J., \& Gaudi, B. S., et al. 2007, ApJ, 660, 781 Bennett, D. P., Bond, I. A., Udalski, A., et al. 2008, ApJ, 684, 663 Bennett, D. P., Batista, V., Bond, I. A., et al. 2014, ApJ, 785, 155 Bennett, D. P., Bhattacharya, A., Anderson, J., et al. 2015, ApJ, 808, 169 Bensby, T., Yee, J. C., Feltzing, S., et al. 2013, A\&A, 549, A147 Bessell, M. S., \& Brett, J. M. 1988, PASP, 100, 1134

Bond, I. A., Bennett, D. P., Sumi, T., et al. 2017, MNRAS, 469, 2434 Chabrier, G. 2003, PASP, 115, 763

Dong, S., DePoy, D. L., Gaudi, B. S., et al. 2006, ApJ, 642, 842

Gaudi, B. S. 1998, ApJ, 506, 533

Gaudi, B. S. 2012, ARA\&A, 50, 411

Gillon, M., Jehin, E., Lederer, S. M., et al. 2016, Nature, 533, 221

Gillon, M., Triaud, A. H. M. J., Demory, B.-O., et al. 2017, Nature, 542, 456

Gould, A. 2000a, ApJ, 535, 928

Gould, A. 2000b, ApJ, 542, 785

Gould, A., Miralda-Escudé, J., Bahcall, J. N. 1994, ApJ, 423, L105

Gould, A., Dong, S., Bennett, D. P., et al. 2010, ApJ, 710, 1800

Han, C., \& Gould, A. 1995, ApJ, 447, 53

Han, C., \& Gould, A. 2003, ApJ, 592, 172

Han, C., Jung, Y. K., Udalski, A., et al. 2013, ApJ, 778, 38

Hwang, K.-H., Choi, J.-Y., Bond, I. A., et al. 2013, ApJ, 778, 55

Hwang, K.-H., Ryu, Y.-H., Kim, H.-W., et al. 2019, AJ, 157, 23

Jung, Y. K., Udalski, A., Gould, A., et al. 2018, AJ, 155, 219

Kervella, P., Thévenin, F., Di Folco, E., \& Ségransan, D. 2004, A\&A, 426, 29

Kim, S.-L., Lee, C.-U., Park, B.-G., et al. 2016, JKAS, 49, 37

Kim, H.-W., Hwang, K.-H., Shvartzvald, Y., et al. 2018a, AAS, submitted [arXiv:1806.07545]

Kim, D.-J., Kim, H.-W., Hwang, K.-H., et al. 2018b, AJ, 155, 76

Kubas, D., Beaulieu, J. P., Bennett, D. P., et al. 2012, A\&A, 540, A78

Laughlin, G., Bodenheimer, P., \& Adams, F. C., 2004, ApJ, 612, L73

Lodato, G., Delgado-Donate, E., \& Clarke, C. J. 2005, MNRAS, 364, L91

Luhman, K. L. 2012, ARA\&A, 50, 65

Nataf, D. M., Gould, A., Fouqué, P., et al. 2013, ApJ, 769, 88

Payne, M. J., \& Lodato, G. 2007, MNRAS, 381, 1597

Shvartzvald, Y., Yee, J. C., Calchi Novati, S., et al. 2017, ApJ, 840, L3

Sumi, T., Udalski, A., Bennett, D. P., et al. 2016, ApJ, 825, 112

Szymański, M. K., Udalski, A., Soszyński, I., et al., 2011, Acta Astron., 61, 83

Tomaney, A. B., \& Crotts, A. P. S. 1996, AJ, 112, 2872

Udalski, A., Szymański, M. K., \& Szymański, G. 2015, Acta Astron., 65, 1

Vandorou, A., Bennett, D. P., Beaulieu, J.-P., et al. 2020, AJ, in press

Yee, J. C., Shvartzvald, Y., Gal-Yam, A., et al. 2012, ApJ, 755, 102

Yee, J. C., Han, C., Gould, A., et al. 2014, ApJ, 790, 14

Yoo, J., DePoy, D. L., Gal-Yam, A., et al. 2004, ApJ, 603, 139

Zhang, X., Zang, W., Udalski, A., et al. 2020, AJ, 159, 116 